\title{
Combining Emerging Sample Preparation Methods, SEM, and TEM Investigations for Microelectronics Device Characterization at Multiple Scales
}

\author{
Pawel Nowakowski*, Cecile Bonifacio, Mary Ray, and Paul Fischione \\ E.A. Fischione Instruments, Inc., Export, PA, USA. \\ *Corresponding author: p_nowakowski@fischione.com
}

Rapid and accurate microelectronics device characterization for failure analyses is currently in demand. Researchers are frequently challenged to produce accurate characterizations quickly from limited sample sizes without material or device background information. The technological advances in microscopy and microanalysis allow these tools to play a large role in the characterization process: transmission and scanning electron microscopes (TEM and SEM) offer constantly improving resolution, analytical detectors (e.g., energy dispersive X-ray spectroscopy [EDS] and electron backscatter diffraction [EBSD]) are increasingly sensitive, and data accusation speeds continue to improve. New analytical techniques, such as transmission Kikuchi diffraction (TKD) have emerged, that extend SEM applications to TEM-prepared specimens. TKD combined with EDS opens new horizons for SEM applications. The advances in analytical tools have occurred simultaneously with the development of new methodologies and instrumentation for sample preparation; the instrumentation not only allows accurate specimen preparation, but also now can assume an active role in analytical investigation. Among a multiplicity of sample preparation techniques, those techniques that are based on ion milling, such as focused ion beam milling (FIB), and broad ion beam (BIB) milling have become the standard in analytical laboratories [1-3]. However, there is an absence of a coherent workflow between SEM and TEM samples preparation and characterization.

We propose a new approach that avoids or dramatically decreases sample preparation-induced artifacts, increases analytical throughput, and allows full-scale analytical observation from micrometer to subnanometer by combining SEM investigation with TEM lamella preparation. We present a solid-state drive (SSD) device with air gaps and a complex structure and composition to which we applied the SEM/TEM workflow. Preparation and investigation of this sample type using a classical approach would make rapid and accurate device and materials characterization a challenge [4, 5]. However, our approach was accomplished in five easy steps:

1. BIB milling of a bulk cross-section sample [SEM Mill, Fischione Instruments] (Figure 1).

2. Delayering of sample to the target layer [SEM Mill, Fischione Instruments] (Figure 2).

3. In situ or ex situ lift out TEM specimen (Figure 3).

4. Concentrated ion beam milling of TEM specimen to decrease specimen thickness and to remove Ga implantation, amorphization, and curtaining [PicoMill ${ }^{\circledR}$ TEM specimen preparation system, Fischione Instruments] (Figure 4).

5. Perform multiscale specimen investigations using SEM, EDS, TKD, TEM, and HRTEM (Figure 5). 
References:

[1] M Schaffer, B Schaffer, and Q Ramasse, Ultramicroscopy 114 (2012), p. 62.

[2] M Simon-Najasek et al., Microelectronics Reliability 54 (2014), p. 1785.

[3] BF Vieweg et al., Ultramicroscopy 113 (2012), p. 165.

[4] M Šikula, T Hrnčír, and P Gounet, Proceedings from the 43rd International Symposium for Testing and Failure Analysis (2017), p. 270.

[5] H-C Hsu et al., Proceedings from the 43rd International Symposium for Testing and Failure Analysis (2017), p. 256.
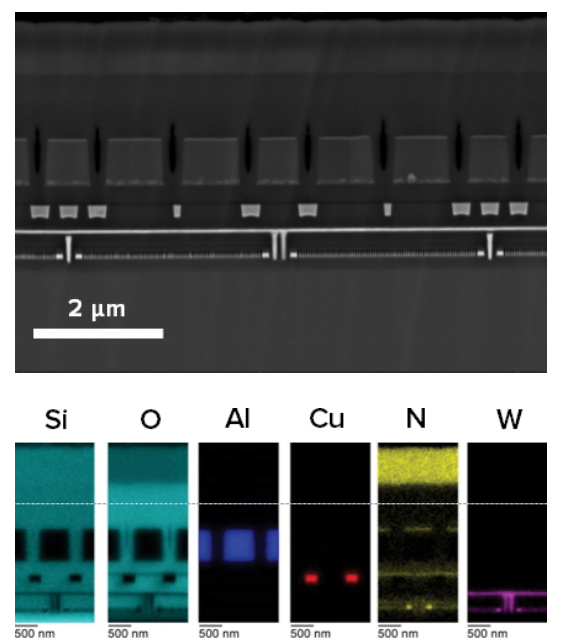

Figure 1. Bulk cross-section SEM image and EDS maps.

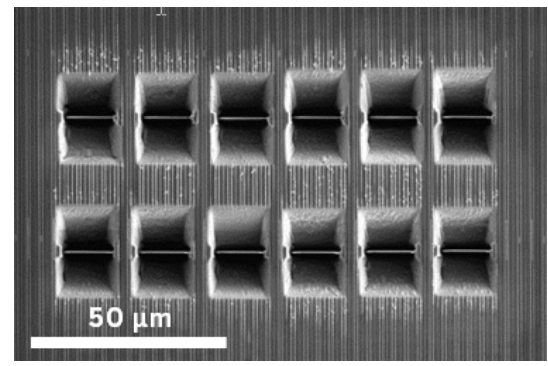

Figure 3. FIB specimen preparation from targeted copper layer.

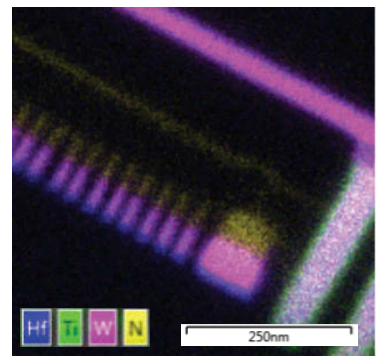

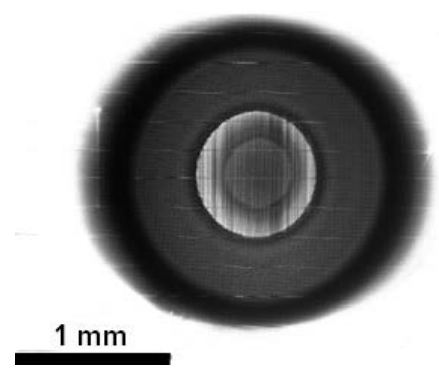

Figure 2. Delayering results to target copper layer: SEM image (left); and EDS maps (right).

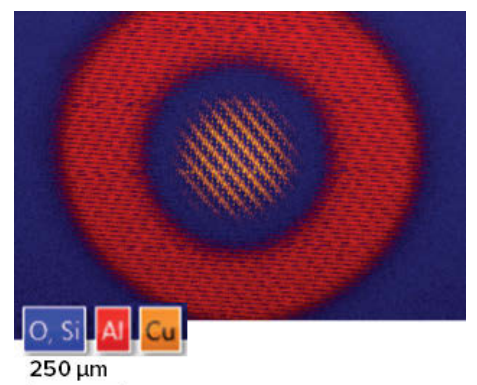

\section{$250 \mu \mathrm{m}$}

Figure 5. Lamella investigation at different scales: SEM EDS elementary map (left) and TEM (right). 